\title{
Transient ischemic attack: management in the emergency department and impact of an outpatient neurovascular clinic
}

\author{
Gregory W. Hosier, MSc*; Stephen J. Phillips, $\mathrm{MD}^{\dagger}$; Steve P. Doucette, MSc ${ }^{\ddagger}$; \\ Kirk D. Magee, MD, MSc ${ }^{\S}$; Gordon J. Gubitz, $\mathrm{MD}^{\dagger}$
}

\section{ABSTRACT}

Objectives: 1) To evaluate whether transient ischemic attack (TIA) management in emergency departments (EDs) of the Nova Scotia Capital District Health Authority followed Canadian Best Practice Recommendations, and 2) to assess the impact of being followed up in a dedicated outpatient neurovascular clinic.

Methods: Retrospective chart review of all patients discharged from EDs in our district from January 1, 2011 to December 31, 2012 with a diagnosis of TIA. Cox proportional hazards models, Kaplan-Meier survival curve, and propensity matched analyses were used to evaluate 90-day mortality and readmission.

Results: Of the 686 patients seen in the ED for TIA, 88.3\% received computed tomography $(\mathrm{CT})$ scanning, $86.3 \%$ received an electrocardiogram (ECG), 35\% received vascular imaging within 24 hours of triage, $36 \%$ were seen in a neurovascular clinic, and $4.2 \%$ experienced stroke, myocardial infarction, or vascular death within 90 days. Rates of antithrombotic use were increased in patients seen in a neurovascular clinic compared to those who were not $(94 \%$ v. $86.3 \%, p<0.0001)$. After adjustment for age, sex, vascular disease risk factors, and stroke symptoms, the risk of readmission for stroke, myocardial infarction, or vascular death was lower for those seen in a neurovascular clinic compared to those who were not (adjusted hazard ratio 0.28; 95\% confidence interval 0.08-0.99, $p=0.048$ ).

Conclusion: The majority of patients in our study were treated with antithrombotic agents in the ED and investigated with CT and ECG within 24 hours; however, vascular imaging and neurovascular clinic follow-up were underutilized. For those with neurovascular clinic follow-up, there was an association with reduced risk of subsequent stroke, myocardial infarction, or vascular death.

\section{RÉSUMÉ}

Objectifs: L'étude visait, d'une part, à examiner si la prise en charge des accidents ischémiques transitoires (AIT) au service des urgences de la région sanitaire Nova Scotia Capital District Health Authority respectait les recommandations canadiennes en matière de pratiques exemplaires, et, d'autre part, à évaluer l'incidence du suivi dans un service de consultations externes, spécialisé en maladies neurovasculaires.

Méthode: Les auteurs ont procédé à un examen rétrospectif des dossiers de tous les patients chez qui avait été posé un diagnostic d'AIT et qui avaient reçu leur congé du SU, dans la région mentionnée, et ce, du $1^{\mathrm{er}}$ janvier 2011 au 31 décembre 2012. La mortalité et le taux de réadmission au bout de 90 jours ont été calculés à l'aide de modèles de risques proportionnels de Cox, de la méthode d'estimation de Kaplan-Meyer et d'analyses appariées de propension.

Résultats: Sur 686 patients examinés au SU pour un AIT, $88,3 \%$ ont été soumis à une tomodensitométrie (TDM); $86,3 \%$, à un ECG; $35 \%$, à un examen par imagerie vasculaire dans les 24 heures suivant le triage et $36 \%$ ont été vus dans un service de consultations spécialisé en maladies neurovasculaires; $4,2 \%$ ont subi un accident vasculaire cérébral (AVC) ou un infarctus du myocarde (IM) ou ont succombé à une maladie vasculaire au bout de 90 jours. Le taux d'utilisation d'antithrombotiques était plus élevé chez les patients qui avaient été vus au service de consultations spécialisé enmaladies neurovasculaires que chez ceux qui n'avaient pas été vus $(94 \%$ contre $86,3 \% ; p<0,0001)$. Après rajustement selon l'âge, le sexe, les facteurs de risque de maladie vasculaire et les symptômes d'AVC, le risque de réadmission pour un AVC, un IM ou une mort par maladie vasculaire était plus faible chez les patients qui avaient été vus au service de consultations spécialisé enmaladies neurovasculaires que chez ceux qui n'avaient pas été vus (rapport de risque rajusté : 0,28; IC à 95\%:0,08-0,99; $p=0,048$ ).

Conclusions: La plupart des patients retenus dans l'étude ont été traités par des antithrombotiques au SU et ont été soumis à une TDM et à un ECG dans les 24 heures; toutefois, le recours aux examens par imagerie vasculaire et au service de consultations spécialisé enmaladies neurovasculaires pour le suivi a fait défaut. Une association a été établie entre le suivi au service de

From the *Faculty of Medicine; †Division of Neurology; ¥Research Methods Unit; §Department of Emergency Medicine, Queen Elizabeth II Health Sciences Centre, Dalhousie University, Halifax, NS.

Correspondence to: Gregory Hosier, Dalhousie University, 5850 College Street, Halifax, NS B3H 4R2; Email: gregory.hosier@dal.ca 
consultations spécialisé enmaladies neurovasculaires et une réduction du risque ultérieur d'AVC, d'IM ou de mort par maladie vasculaire.
Keywords: TIA, transient ischemic attack, emergency department, stroke management, neurovascular clinic, stroke prevention clinic

\section{INTRODUCTION}

Transient ischemic attacks (TIAs) warn of increased risk of stroke, other vascular events, and death. ${ }^{1}$ The risk of recurrent stroke after TIA is $5 \%-20 \%$ within 90 days, with about half of all recurrent strokes occurring in the first two days following initial symptom onset. ${ }^{1-4}$ Early assessment in a dedicated neurovascular clinic and immediate initiation of medical and surgical therapy have been shown to reduce the risk of major stroke after TIA by up to $80 \% .^{5}$

Patients with TIA often present to the emergency department (ED) for initial evaluation. The current Canadian Best Practice Recommendations for stroke (and TIA) stipulate that any patient who presents to the ED with a suspected recent stroke or TIA undergo assessment within 24 hours that includes brain imaging, noninvasive vascular imaging (for suspected carotid territory events), electrocardiogram (ECG), initiation or modification of secondary prevention therapy (antihypertensive, lipid-lowering, glucose-lowering, or anticoagulation therapy), and referral to a designated stroke prevention clinic for risk factor assessment. ${ }^{6}$ A nation-wide audit of stroke and TIA care in Canada (Quality of Stroke Care 2011) showed that many patients with TIA do not receive timely investigations and treatment. ${ }^{7}$ We were interested in obtaining a snapshot of the care processes for patients who received a diagnosis of TIA in the EDs of the Capital District Health Authority in Nova Scotia, and to determine the impact of being seen in a dedicated neurovascular clinic on patient outcomes.

\section{METHODS}

\section{Study design and subjects}

This study was approved by the health system Research Ethics Board. This was a historical cohort study of all patients discharged from the EDs during the 2-year period of January 1, 2011 through December 31, 2012 with a diagnosis of TIA as identified using our ED information system. This regional health authority system serves a population of approximately 400,000, and its four EDs have a combined annual census of approximately 160,000 patients. The ED information system contains the diagnosis at discharge recorded by the attending ED physician for all ED visits in the system. We followed the health records of all identified patients who met study criteria for a 90-day period following the index TIA event. TIA was defined as a sudden, focal neurological deficit of presumed vascular origin lasting $<24$ hours. Data were not used if a medical record indicated the patient did not want their information used for research purposes. The Division of Neurology, based in the Queen Elizabeth II Health Sciences Centre (the largest hospital in Nova Scotia) provides a 24/7 emergency neurology consultation service, either in person or by telephone. A daily (Monday through Friday) neurovascular clinic, operated by a stroke neurologist and neurology nurse practitioner, evaluates patients referred by emergency (and other) physicians. The standard of care is for patients with suspected TIA or stroke to be seen in the ED by the Neurology services during the same or next day. Our policy is to initiate investigations and start or modify treatment in the ED, and discharge the patient home with arrangements for fasting blood tests and outpatient follow-up in the neurovascular clinic. Patients may be admitted to the Acute Stroke Inpatient Unit if judged necessary by the on-call neurologist, for example, if there has been more than one TIA over the preceding 24 hours, or if there is comorbidity requiring hospitalization, or residence remote from the hospital, or probable or definite severe symptomatic carotid stenosis.

\section{Data collection}

An initial search of the ED information system identified patients diagnosed with TIA or transient ischemic attack within our time frame of interest. One person conducted the chart abstraction without a priori knowledge of the content of the records. Source documents included emergency physician notes, paramedic notes, registered nurse notes, consultation notes, outpatient clinic notes, inpatient discharge notes, and death summaries. The health system databases do 
not include information related to clinical visits outside of affiliated facilities, such as family doctors' offices. Abstracted data included patient demographics, medical history, care provided throughout the hospital stay, inpatient and outpatient investigations, medication prescriptions, discharge status, hospital readmission within 90 days of the index TIA, and death within 90 days of the index TIA if recorded in the health system clinical databases. Data for clinical variables (history of smoking, hypertension, etc.) not recorded in any of the clinical information were coded as negative.

\section{Outcomes}

Process outcomes were the proportion of patients referred to the neurovascular clinic, the proportion of TIA patients seen in the clinic, the proportion of TIA patients that received guide-line recommended assessment (brain CT or magnetic resonance imaging [MRI], carotid imaging, fasting blood tests, ECG, and international normalized ratio [INR]) for those seen in ED only and those seen in the ED and the neurovascular clinic, and the proportion of patients that were on antiplatelet, anticoagulation, antihypertensive, lipid-lowering, or glucose-lowering therapy after being seen in the ED and after follow-up in the neurovascular clinic. Our main clinical outcome was the comparison of readmission rates within 90 days of index TIA for ischemic stroke, myocardial infarction, or vascular death for patients seen in the neurovascular clinic versus those who were not.

\section{Analysis}

Population characteristics were summarized as means with standard deviation for continuous data and frequencies with percentage for categorical data. For process outcomes (investigations, treatments), descriptive analyses were conducted. Kaplan-Meier survival analysis and log-rank testing were used to compare clinical outcomes of patients seen in the neurovascular clinic with those who were not. Adjusted Cox proportional hazard models were used to compare time to readmission or death from ischemic stroke or myocardial infarction in those seen in the neurovascular clinic compared to those not who were not. The proportionality assumption was not violated in any of the models considered. Parallel curves resulted in the analysis of the survival function versus time and the $\log$ (-log survival) versus $\log$ of the survival time. A propensity analysis was used to account for potential confounding and selection biases. The propensity for patients seen in the neurovascular clinic was developed using multivariable logistic regression. Variables included in this derivation were age, sex, location, risk factors, medication use, and symptoms. A greedy matching procedure matched propensity scores to five decimals initially. ${ }^{8}$ This match continued reducing the number of decimals to one. If no match existed at one decimal place, the patient was not used for analysis. Statistical analysis was performed using SAS version 9.4 (Cary, NC).

\section{RESULTS}

An initial search of the ED information system identified 697 patients with a discharge diagnosis of TIA. After review of the records, 11 patients were identified that were mislabeled in the information system and did not in fact have an ED discharge diagnosis of TIA. Of the remaining 686 patients, $33 \%$ were seen by Neurology in the $\mathrm{ED}, 51 \%$ were referred to the neurovascular clinic, and $36 \%$ were actually seen in the clinic (Figure 1). Table 1 compares the clinical characteristics of patients seen in the ED (and not the neurovascular clinic) with those patients seen in the ED and the neurovascular clinic. After propensity matching, there were 215 patients who were seen in the ED only and 215 patients seen in both the ED and neurovascular clinic with statistically similar baseline characteristics (Table 2). Median time from TIA event to triage was 2 hours (1.1-6.6 hours interquartile range $[\mathrm{IQR}])$. The ED discharge diagnosis of TIA agreed with the final diagnosis after being seen in the neurovascular clinic in $86.4 \%$ of cases. Of the patients who received an alternative diagnosis from the neurovascular clinic, migraine (24\%), syncope/presyncope

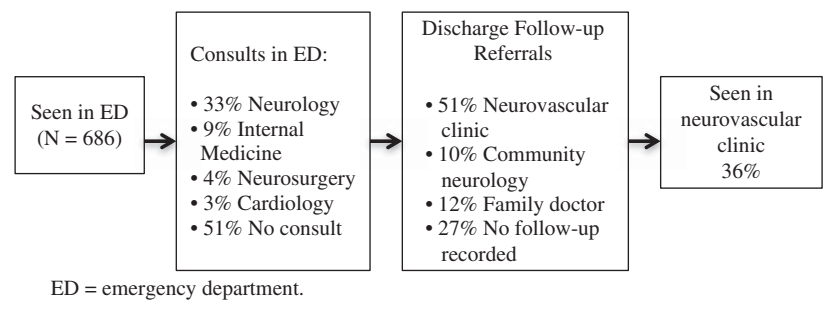

Figure 1. Care flow chart showing consults requested in the emergency department, follow-up referrals, and patients seen in the neurovascular clinic as a percentage of the total study population ( $\mathrm{N}=686$ ). Consults in ED and discharge referrals were not mutually exclusive, meaning patients who were seen by Neurology in the ED may also have received a discharge referral to and been seen in the neurovascular clinic. ED = emergency department. 


\begin{tabular}{|c|c|c|c|}
\hline Variable & No clinic & Clinic & $p$-value \\
\hline Age, yr, mean (SD) & $72.5(13.7)$ & $68.8(11.3)$ & 0.0002 \\
\hline Male sex & $224(51.5)$ & $136(55.3)$ & 0.38 \\
\hline \multicolumn{4}{|l|}{ Presenting emergency site } \\
\hline Site 1 & $193(44.4)$ & $129(52.4)$ & $<0.0001$ \\
\hline Site 2 & $133(30.6)$ & $52(21.1)$ & \\
\hline Site 3 & $65(14.9)$ & $58(23.6)$ & \\
\hline Site 4 & $44(10.1)$ & $7(2.9)$ & \\
\hline \multicolumn{4}{|l|}{ Risk factors } \\
\hline Hypertension & $306(71.8)$ & $161(66.3)$ & 0.14 \\
\hline Diabetes & $112(26.3)$ & $62(25.4)$ & 0.85 \\
\hline Previous TIA & $123(28.5)$ & 72 (29.3) & 0.86 \\
\hline Dyslipidemia & $221(52.4)$ & 139 (57.9) & 0.19 \\
\hline Smoking & $50(12.9)$ & 48 (19.8) & 0.02 \\
\hline Atrial fibrillation & $54(12.5)$ & $23(9.4)$ & 0.26 \\
\hline \multicolumn{4}{|l|}{ Medications } \\
\hline Antihypertensive & $288(66.2)$ & $150(61.0)$ & 0.18 \\
\hline Antithrombotic & $251(57.7)$ & $123(50)$ & 0.05 \\
\hline ASA & $172(39.5)$ & $83(33.7)$ & 0.14 \\
\hline Clopidogrel & $53(12.2)$ & $29(11.8)$ & 0.9 \\
\hline Warfarin & $41(9.4)$ & $9(3.7)$ & 0.006 \\
\hline Dipyridamole + ASA & 7 (1.6) & $3(1.2)$ & 1 \\
\hline Dabigatran & $10(2.3)$ & $2(0.8)$ & 0.23 \\
\hline Rivaroxaban & $0(0)$ & $1(0.4)$ & 0.36 \\
\hline Apixaban & $2(0.5)$ & $0(0)$ & 0.54 \\
\hline Lipid-lowering & $210(48.3)$ & $122(49.6)$ & 0.75 \\
\hline Glucose-lowering & $101(23.2)$ & $58(23.6)$ & 0.93 \\
\hline \multicolumn{4}{|l|}{ TIA symptoms* } \\
\hline Unilateral weakness & $218(50.4)$ & $117(47.6)$ & 0.52 \\
\hline Speech disturbance & $177(40.8)$ & $113(45.9)$ & 0.2 \\
\hline Sensory loss/change & $153(35.3)$ & $99(40.2)$ & 0.22 \\
\hline Vertigo & $19(4.4)$ & $16(6.5)$ & 0.28 \\
\hline Confusion & $100(23)$ & $34(13.8)$ & 0.004 \\
\hline Dizziness $^{\dagger}$ & $81(18.7)$ & $51(20.7)$ & 0.55 \\
\hline Headache & 73 (16.9) & $59(24)$ & 0.03 \\
\hline Gait disturbance & $65(15)$ & $41(16.7)$ & 0.58 \\
\hline Vision changes & 75 (17.3) & $62(25.2)$ & 0.02 \\
\hline \multicolumn{4}{|l|}{ TIA duration } \\
\hline$<10 \min$ & $60(14)$ & 45 (18.3) & 0.77 \\
\hline $11-60 \mathrm{~min}$ & $243(56.6)$ & $122(49.6)$ & \\
\hline$>60 \min$ & $126(29.4)$ & $79(32.1)$ & \\
\hline
\end{tabular}

(7\%), seizure (6\%), and brain neoplasm (4\%) were the most common alternative diagnoses. Patients who did not receive a final diagnosis of TIA after being seen in the neurovascular clinic were included in all subsequent analyses because, at the time of their presentation, they were being managed as cases of TIA. Median

\begin{tabular}{|c|c|c|c|}
\hline Variable & No clinic & Clinic & $p$-value \\
\hline Age, yr, mean (SD) & $69.4(14.6)$ & $69.0(11.3)$ & 0.74 \\
\hline Male sex & $114(53 \%)$ & $118(54.9 \%)$ & 0.77 \\
\hline \multicolumn{4}{|l|}{ Presenting ED } \\
\hline Site 1 & $124(57.7 \%)$ & 117 (54.4\%) & 0.86 \\
\hline Site 2 & $46(21.4 \%)$ & $48(22.3 \%)$ & \\
\hline Site 3 & $41(19.1 \%)$ & $44(20.5 \%)$ & \\
\hline Site 4 & $4(1.9 \%)$ & $6(2.8 \%)$ & \\
\hline \multicolumn{4}{|l|}{ Risk factors } \\
\hline Hypertension & $148(68.8 \%)$ & $143(66.5 \%)$ & 0.73 \\
\hline Diabetes & $58(27 \%)$ & $56(26.1 \%)$ & 0.91 \\
\hline Previous TIA & $66(30.7 \%)$ & $63(29.3 \%)$ & 0.83 \\
\hline Dyslipidemia & $128(59.5 \%)$ & $121(56.3 \%)$ & 0.56 \\
\hline Smoking & 35 (16.3\%) & $38(17.7 \%)$ & 0.8 \\
\hline Atrial fibrillation & $18(8.4 \%)$ & $19(8.8 \%)$ & 1 \\
\hline \multicolumn{4}{|l|}{ Medications } \\
\hline Antihypertensive & $138(64.2 \%)$ & $133(61.9 \%)$ & 0.69 \\
\hline Antithrombotic & $107(49.8 \%)$ & $111(51.6 \%)$ & 0.77 \\
\hline ASA & $67(31.2 \%)$ & 75 (34.9\%) & 0.47 \\
\hline Clopidogrel & $25(11.6 \%)$ & $27(12.6 \%)$ & 0.88 \\
\hline Warfarin & $9(4.2 \%)$ & $8(3.7 \%)$ & 1 \\
\hline Dipyridamole + ASA & $4(1.9 \%)$ & $3(1.4 \%)$ & 1 \\
\hline Dabigatran & $3(1.4 \%)$ & $2(0.9 \%)$ & 1 \\
\hline Rivaroxaban & $0(0 \%)$ & $0(0 \%)$ & 1 \\
\hline Apixaban & $1(0.5 \%)$ & $0(0 \%)$ & 1 \\
\hline Lipid-lowering & $115(53.5 \%)$ & $106(49.3 \%)$ & 0.44 \\
\hline Glucose-lowering & $51(23.7 \%)$ & $52(24.2 \%)$ & 1 \\
\hline \multicolumn{4}{|l|}{ TIA symptoms* } \\
\hline Unilateral weakness & $99(46.1 \%)$ & $103(47.9 \%)$ & 0.7 \\
\hline Speech disturbance & $91(42.3 \%)$ & $97(45.1 \%)$ & 0.63 \\
\hline Sensory loss/change & $84(39.1 \%)$ & $85(39.5 \%)$ & 0.92 \\
\hline Vertigo & $12(5.6 \%)$ & $14(6.5 \%)$ & 0.84 \\
\hline Confusion & $30(14 \%)$ & $30(14 \%)$ & 1 \\
\hline Dizziness $^{\dagger}$ & $48(22.3 \%)$ & $42(19.5 \%)$ & 0.55 \\
\hline Headache & $41(19.1 \%)$ & $45(20.9 \%)$ & 0.72 \\
\hline Gait disturbance & $36(16.7 \%)$ & $36(16.7 \%)$ & 1 \\
\hline Vision changes & $52(24.2 \%)$ & $51(23.7 \%)$ & 1 \\
\hline \multicolumn{4}{|l|}{ TIA duration } \\
\hline$<10 \min$ & $31(14.4 \%)$ & $43(20 \%)$ & 0.57 \\
\hline $11-60 \mathrm{~min}$ & $119(55.4 \%)$ & $103(47.9 \%)$ & \\
\hline$>60 \min$ & $65(30.2 \%)$ & $69(32.1 \%)$ & \\
\hline \multicolumn{4}{|c|}{$\begin{array}{l}\text { *Multiple symptoms per patient. } \\
\text { 'Dizziness may be the same as vertigo. } \\
\text { ASA = acetylsalicylic acid; ED = emergency department; TIA = transient ischemic } \\
\text { attack. }\end{array}$} \\
\hline
\end{tabular}

time from TIA to time seen in the neurovacular clinic was 32 days (10-60 days IQR) for all patients and 22 days (9-52 days IQR) for those not seen by Neurology in the ED.

In the propensity matched groups, there was no difference in the rates of ECG or INR obtainment in 
patients seen in the ED only compared to those seen in the ED and neurovascular clinic within 24 hours, 7 days, 30 days, and 90 days of triage (Table 3). There was increased rates of CT scanning in those seen in the neurovascular clinic within 7 days $(95.4 \%)$ compared to those not seen in the neurovascular clinic (90.2\%), and this trend continued at each time point thereafter (Table 3). There were increased rates of vascular imaging with Doppler ultrasound (US) in those seen in the neurovascular clinic versus those who were not seen at 24 hours, 7 days, 30 days, and 90 days following triage (Table 3). There was no difference in rates of magnetic resonance angiography and computed tomography angiography use in patients seen in the neurovascular clinic versus those who were not seen (Table 3). Fasting blood work was obtained in $60.5 \%$ of patients seen in the ED and $77.7 \%$ of patients who were seen in both the ED and the neurovascular clinic. Among all patients $(\mathrm{N}=686)$, ECG was obtained in $87.5 \%$, INR in $59.5 \%$, CT in $92.9 \%$, MRI in $18.7 \%$, Doppler US in $56.6 \%$, magnetic resonance angiography in $16.8 \%$, computed tomography angiography in $7.6 \%$, and fasting blood work in $61.6 \%$ within 90 days of triage in the ED.

In the propensity matched groups, $86.3 \%$ of patients seen in the ED (and not neurovascular clinic) were on antithrombotic therapy (59\% received new or modified antithrombotic medication), compared to $94 \%$ of patients seen in both the ED and the neurovascular clinic $(76 \%$ received new or modified antithrombotic medication; Table 4). Of patients with atrial fibrillation (newly or previously diagnosed), $47.6 \%$ were on anticoagulant therapy after being seen in the ED, and this increased to $85.7 \%$ in those seen in the neurovascular clinic (Table 4). Of the 19 patients who were candidates for carotid endarterectomy, $57.9 \%$ received carotid endarterectomy within 2 weeks after the index TIA.

Of the 686 patients seen in the ED, $4.2 \%$ experienced subsequent ischemic stroke, myocardial ischemia, or vascular death within 90 days. After adjustment for age, sex, presence of vascular disease risk factor, and stroke symptoms, the risk of readmission for stroke, myocardial infarction, or vascular death was lower for those seen in the neurovascular clinic (adjusted hazard ratio [HR] 0.28; 95\% confidence interval [CI] 0.08-0.99, $p=0.048)$. The risk of readmission or death from ischemic stroke or myocardial infarction at 90 days in the propensity matched cohort was significantly lower in patients who were seen in the neurovascular clinic

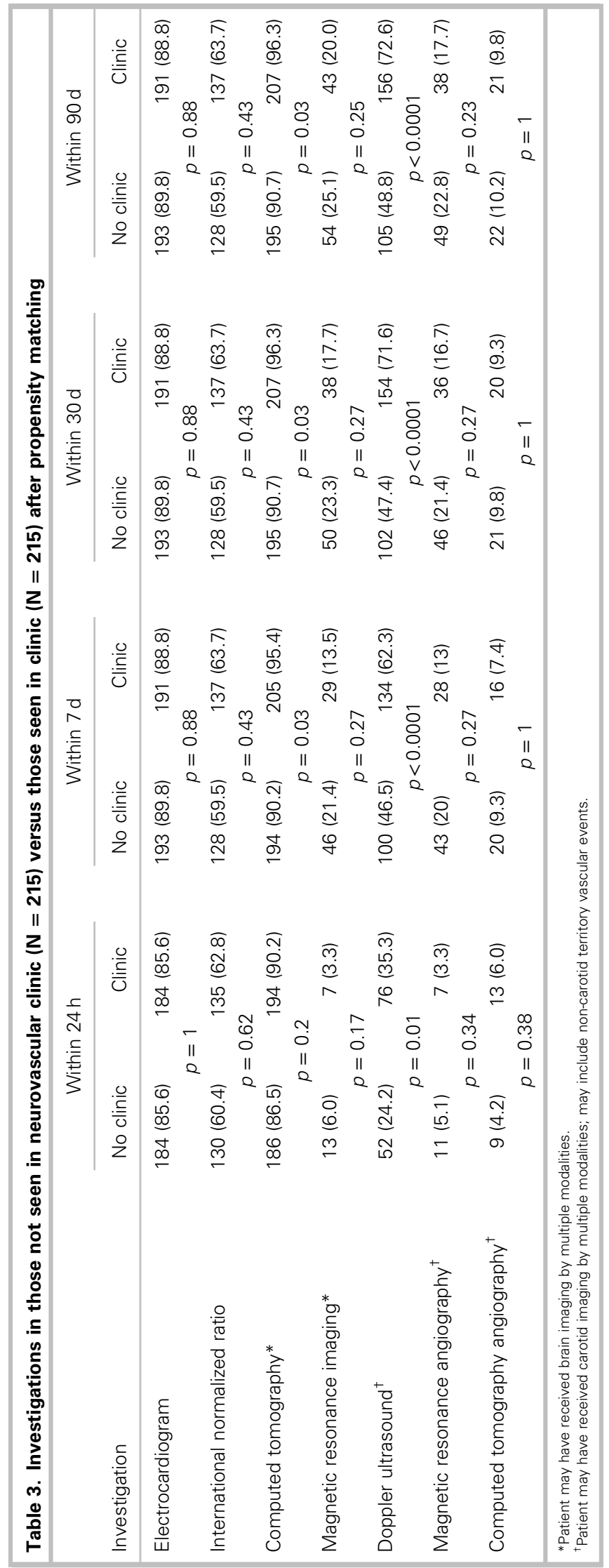


compared to patients not seen (Table 5 and Figure 2; HR $0.18,95 \%$ CI $0.04-0.8, p=0.02)$. In the matched cohort, two patients were denied neurovascular clinic services because they were palliative. Both of these patients remained event-free for the study period.

\section{DISCUSSION}

The majority of patients diagnosed with TIA in our cohort were treated with antithrombotic agents in the

\begin{tabular}{|c|c|c|c|}
\hline \multicolumn{4}{|c|}{ No. (and \%) of patients } \\
\hline Characteristic & $\begin{array}{l}\text { No clinic } \\
(N=215)\end{array}$ & $\begin{array}{c}\text { Clinic } \\
(N=215)\end{array}$ & $p$-value \\
\hline Antithrombotic & $166(86.3)$ & $202(94.0)$ & $<0.0001$ \\
\hline Antihypertensive & $142(66.1)$ & $153(71.2)$ & 0.3 \\
\hline Lipid-lowering & $122(56.7)$ & $159(74.0)$ & 0.0003 \\
\hline Glucose-lowering & $52(24.2)$ & $55(25.6)$ & 0.82 \\
\hline $\begin{array}{l}\text { Anticoagulant if atrial } \\
\text { fibrillation }\end{array}$ & $10 / 21(47.6)$ & $\begin{array}{l}18 / 21 \\
(85.7)\end{array}$ & $<0.0001$ \\
\hline $\begin{array}{l}\text { Carotid endarterectomy } \\
\text { within } 14 \text { days }\end{array}$ & $5(2.3)$ & $4(1.9)$ & 1 \\
\hline
\end{tabular}

ED and investigated with brain imaging and ECG within 24 hours of triage, but only about half of the patients were referred to the neurovascular clinic. The number of patients who experienced subsequent ischemic stroke, myocardial infarction, or vascular death within 90 days was low, at $4.2 \%$. Although management and outcomes for all patients seen with TIA in the ED were generally favourable, those seen in

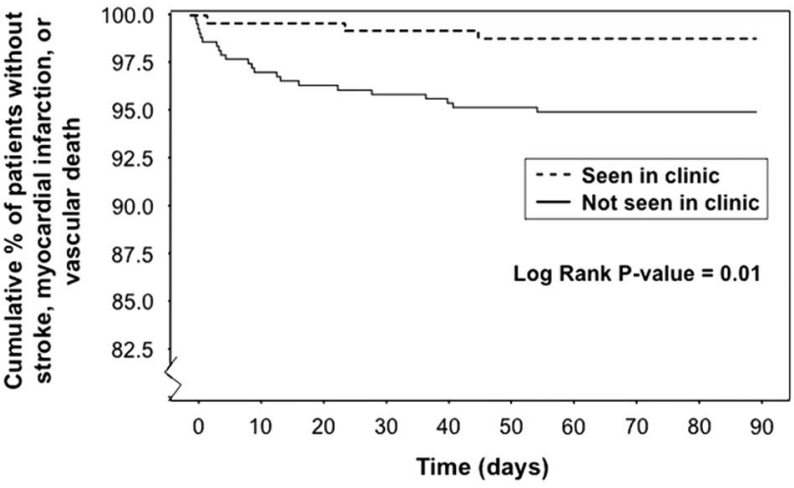

Figure 2. Fewer patients readmitted with ischemic stroke or myocardial infarction, or died from ischemic stroke or myocardial infarction, within 90 days after index TIA when seen in the neurovascular clinic $(N=215)$ compared to those who were not seen in the neurovascular clinic $(\mathrm{N}=215)$ after propensity matching.

\begin{tabular}{|c|c|c|c|c|c|c|c|c|}
\hline \multirow[b]{2}{*}{ Outcomes } & \multicolumn{2}{|c|}{ Within $2 d$} & \multicolumn{2}{|c|}{ Within 7d } & \multicolumn{2}{|c|}{ Within $30 \mathrm{~d}$} & \multicolumn{2}{|c|}{ Within $90 \mathrm{~d}$} \\
\hline & No clinic & Clinic & No clinic & Clinic & No clinic & Clinic & No clinic & Clinic \\
\hline \multirow[t]{2}{*}{ Return to ED reason: Transient ischemic attack } & $2(0.9)$ & $5(2.3)$ & $9(4.2)$ & $9(4.2)$ & $14(6.5)$ & $16(7.4)$ & $15(7)$ & $22(10.2)$ \\
\hline & \multicolumn{2}{|c|}{$p=0.25$} & \multicolumn{2}{|c|}{$p=1$} & \multicolumn{2}{|c|}{$p=0.85$} & \multicolumn{2}{|c|}{$p=0.3$} \\
\hline \multirow[t]{2}{*}{ Stroke } & $4(1.9)$ & $0(0)$ & $6(2.8)$ & $0(0)$ & $8(3.7)$ & $1(0.5)$ & $9(4.2)$ & $2(0.9)$ \\
\hline & \multicolumn{2}{|c|}{$p=0.12$} & \multicolumn{2}{|c|}{$p=0.03$} & \multicolumn{2}{|c|}{$p=0.04$} & \multicolumn{2}{|c|}{$p=0.06$} \\
\hline \multirow[t]{2}{*}{ Intracranial hemorrhage } & $0(0)$ & $1(0.5)$ & $0(0)$ & $1(0.5)$ & $0(0)$ & $1(0.5)$ & $1(0.5)$ & $1(0.5)$ \\
\hline & \multicolumn{2}{|c|}{$p=1$} & \multicolumn{2}{|c|}{$p=1$} & \multicolumn{2}{|c|}{$p=1$} & \multicolumn{2}{|c|}{$p=1$} \\
\hline \multirow[t]{2}{*}{ Myocardial infarction } & $0(0)$ & $0(0)$ & $0(0)$ & $0(0)$ & $0(0)$ & $0(0)$ & $0(0)$ & $0(0)$ \\
\hline & \multicolumn{2}{|c|}{$p=1$} & \multicolumn{2}{|c|}{$p=1$} & \multicolumn{2}{|c|}{$p=1$} & \multicolumn{2}{|c|}{$p=1$} \\
\hline \multirow[t]{2}{*}{ Other } & $0(0)$ & $1(0.5)$ & $1(0.5)$ & $4(1.9)$ & $18(8.4)$ & $13(6)$ & $26(12.1)$ & $24(11.2)$ \\
\hline & \multicolumn{2}{|c|}{$p=1$} & \multicolumn{2}{|c|}{$p=0.37$} & \multicolumn{2}{|c|}{$p=0.57$} & \multicolumn{2}{|c|}{$p=0.95$} \\
\hline \multirow[t]{2}{*}{ Cause of death: Stroke } & $0(0)$ & $0(0)$ & $0(0)$ & $0(0)$ & $1(0.5)$ & $0(0)$ & $2(0.9)$ & $1(0.5)$ \\
\hline & \multicolumn{2}{|c|}{$p=1$} & \multicolumn{2}{|c|}{$p=1$} & \multicolumn{2}{|c|}{$p=1$} & \multicolumn{2}{|c|}{$p=1$} \\
\hline Intracranial hemorrhage & $0(0)$ & $0(0)$ & $1(0.5)$ & $0(0)$ & $1(0.5)$ & $0(0)$ & $2(0.9)$ & $0(0)$ \\
\hline & $p=$ & & $p=$ & & $p$ & & $p=$ & \\
\hline Cancer & $0(0)$ & $0(0)$ & $1(0.5)$ & $0(0)$ & $1(0.5)$ & $0(0)$ & $1(0.5)$ & $0(0)$ \\
\hline & $p=$ & & $p=$ & & $p$ & & & \\
\hline Myocardial infarction & $0(0)$ & $0(0)$ & $0(0)$ & $0(0)$ & $1(0.5)$ & $0(0)$ & $1(0.5)$ & $0(0)$ \\
\hline & $p=$ & & $p=$ & & $p$ & & $p$ & \\
\hline
\end{tabular}


the neurovascular clinic had lower rates of readmission for stroke, myocardial infarction, or vascular death (adjusted HR 0.28; 95\% CI 0.08-0.99, $p=0.048$ ).

There is growing evidence that access to outpatient clinics aimed at risk factor management improves rates of investigations, treatments, and outcomes of patients with TIA. ${ }^{5,9,10}$ Using data from 12 specialized stroke centers in Ontario between 2003 and 2008, Webster et al. (2011) found that referral of patients with ischemic stroke or TIA to a designated neurovascular clinic was associated with a $29 \%$ reduction in 1-year mortality (HR, 0.71; 95\% CI, 0.65-0.84). Similar to our study, Webster et al. (2011) found that only $47 \%$ of the study population was referred to the neurovascular clinic in follow-up, indicating that outpatient neurovascular clinics may be underutilized. ${ }^{9}$ Although we did not specifically look at reasons for non-referral, some reasons may include referral to a family doctor or community neurologist, failure to document a referral, and lack of awareness of the neurovascular clinic by the ED physician. Reasons for not being seen after referral may include patient noncompliance and miscommunication. The mechanisms underlying the improved outcomes seen with care provided by the neurovascular clinic are likely multifactorial and may include more timely investigations, initiation of appropriate medical therapy for secondary stroke prevention, and access to other professionals with expertise in stroke care. This is supported by our findings that rates of antithrombotic use, lipid-lowering agents, anticoagulants in those with atrial fibrillation, fasting blood tests, CT scan rates, and Doppler US were increased in patients seen in the neurovascular clinic compared to those not seen.

Interestingly, there was morbidity/mortality benefit in the first 7 days following the index TIA among patients who were seen in the neurovascular clinic compared to those who were not, while the median time to being seen in the neurovascular clinic was 32 days. This suggests that there were systemic differences between the two groups. To limit such differences, we used propensity matching, which generated subpopulations with statistically similar baseline characteristics for those seen in ED-only patients, and those seen in both the ED and the neurovascular clinic. A protective effect of attending at the neurovascular clinic was still apparent after this adjustment. The median time to being seen in the neurovascular clinic for patients not seen by Neurology in the ED was 22 days (9-52 days IQR) compared to 32 days (10-60 days IQR) for those not seen by Neurology in the ED. This reflects an effort by practitioners in the neurovascular clinic to see patients not assessed by Neurology in the ED sooner. It is also standard practice for practitioners in the neurovascular clinic to call in prescriptions for secondary stroke medications and request imaging studies and blood work for these patients before being seen in the clinic. Together, these factors may help explain the early protective effect for patients who would later go on to be seen in the neurovascular clinic for follow-up.

It is well known that timely investigations and use of medications for secondary stroke risk factor management improve stroke outcomes. Our rates of CT (88.3\%) and ECG (86.3\%) investigations within 24 hours were similar to those reported by Perry et al. (2013) and compare favourably to those reported in the Quality of Stroke Care in Canada 2011 report. $^{7,11}$ Rates of antithrombotic, antihypertensive, and lipid-lowering agents were similar in this study compared to the Quality of Stroke Care 2011 report and other studies. ${ }^{7,11}$ Vascular imaging was obtained in approximately $55 \%$ of patients. Rates of vascular imaging in other studies are also low, ranging from $15 \%-42 \% .{ }^{4,12,13}$ Approximately $15 \%$ of all TIAs are due to large artery atherosclerosis. ${ }^{14,15}$ Timely vascular imaging is needed to identify patients with $50 \%-99 \%$ carotid stenosis so that they can receive carotid endarterectomy surgery within 2 weeks of symptom onset, the time frame in which the greatest benefit is seen. ${ }^{16,17}$ New to the updated Canadian Best Recommendations (2014) is the recommendation to use CT angiography at the time of brain CT as the first-line vascular imaging method. ${ }^{6}$ For patients in whom a cardioembolic mechanism is suspected, but the ECG does not show atrial fibrillation, prolonged ECG monitoring is recommended in selected patients (older patients with recent embolic stroke of undetermined source who are potential candidates for anticoagulation therapy) for the detection of paroxysmal atrial fibrillation., ${ }^{6,18}$ Our results indicate the need for increased investigation into stroke mechanism, specifically carotid imaging, in patients presenting with TIA.

Distinguishing between TIA and ischemic stroke in the acute setting of the ED can be challenging. The traditional definition of TIA is a sudden, focal neurological deficit of presumed vascular origin lasting $<24$ hours. Decisions regarding thrombolytic therapy need to be made within minutes of presentation to the ED. A 24-hour definition for TIA encourages delay in administration of acute thrombolytic therapy. With advances in MRI, up to three-quarters of TIAs defined 
by the traditional time-based definition have been found to have evidence of tissue damage. ${ }^{19}$ This has sparked a shift towards use of a tissue-based definition of TIA. However, timely access to MRI and expert interpretation is not always available, and MRI is not cost-effective compared to $\mathrm{CT} .{ }^{20}$ It also remains to be seen whether use of plasminogen activator in patients with recent prior clinical symptoms lasting $<24$ hours associated with small MRI lesions is safe and effective. For these reasons, a tissue-based definition of TIA has yet to be widely endorsed. Furthermore, from a stroke prevention perspective, the risk of major stroke following a minor event is the same for TIA and mild stroke. ${ }^{21}$ We found that $4.2 \%$ patients diagnosed as TIA in the ED were found to have ischemic culprit lesions identified on CT or MRI at the time of presentation or thereafter. Including or excluding these patients in analyses did not change our results. The majority of TIAs in our study lasted $<1$ hour, and this is true in other studies. ${ }^{11,22}$ A more practical definition of TIA in the ED seems to be a sudden-onset, focal neurological deficit lasting less than 1 hour.

Traditionally, diagnostic accuracy of TIA and minor stroke in the ED has been somewhat low, ranging from approximately $50 \%-64 \% .^{23-25}$ In our study, the discharge diagnosis of TIA agreed with the diagnosis after being seen in the neurovascular clinic in $86.4 \%$ of patients. Including or excluding these patients did not change our results. The most frequent alternative TIA mimics were migraine, syncope/presyncope, seizure, and brain neoplasm. It is possible that consultation of Neurology in the ED for patients with unclear presentation contributed to high rates of diagnostic accuracy in the current study.

\section{Limitations}

The health system databases do not include information pertaining to clinical visits outside of this health system (for example, independent family doctors' offices). It is therefore possible that our main clinical outcome of readmission for stroke, myocardial infarction, or death from stroke or myocardial infarction may have underestimated the actual recurrence rate of stroke, myocardial infarction, or death from stroke or myocardial infarction. Unfortunately, we are not able to estimate how many patients may have presented to a facility outside of our health system. However, we suspect that it would be low, because it is standard practice for the family doctor to send any patients with symptoms of stroke or myocardial infarction directly to the nearest $\mathrm{ED}$, which would presumably be the same ED to which the patient presented initially.

It is possible that a proportion of patients were not referred to the neurovascular clinic because they were palliative or had severe dementia and instead were referred to their family physician for follow-up care. This may have biased the rates of stroke recurrence, myocardial infarction, and death to be higher in those not seen in the neurovascular clinic. In the matched cohort, we identified only two patients who were denied services in the neurovascular clinic because they were palliative. Both of these patients remained event free. However, palliative status for all patients may not have been documented in the clinical databases. Furthermore, we did not analyse presence of dementia in our patient cohort.

A potential confounding factor in our study was that patients who were referred to the neurovascular clinic could have had an adverse event that prevented them from being seen in follow-up. Arguing against this is accrued data (not shown) that rates of adverse events for those referred (but not seen in the clinic) were not different than rates of adverse events for patients seen in the clinic.

Finally, because this was a retrospective chart review, it is not possible to discern whether all patient risk factors, neurological deficits, medications, or the time of TIA event were fully and accurately recorded. By design, we attempted to minimize abstraction bias, with one person conducting the chart abstraction without a priori knowledge of the content of the records, and outcome data were based on objective documentation.

\section{CONCLUSION}

Our results support the use of outpatient clinics aimed at management of vascular disease risk factors as one way to improve outcomes of patients with TIA. Use of CT, ECG, and antiplatelet therapy in the ED was high, but there remains a need for more rapid vascular imaging, as well as more aggressive medical management, including oral anticoagulant therapy for those with evidence of atrial fibrillation in the ED after a TIA, in an effort to prevent disabling stroke or death.

Competing interests: None declared. 


\section{REFERENCES}

1. Furie KL, Kasner SE, Adams RJ, et al. Guidelines for the prevention of stroke in patients with stroke or transient ischemic attack: a guideline for healthcare professionals from the American Heart Association/American Stroke Association. Stroke 2011;42(1):227-76, doi:10.1161/ STR.0b013e3181f7d043.

2. Johnston SC, Gress DR, Browner WS, et al. Short-term prognosis after emergency department diagnosis of TIA. ZAMA 2000;284(22):2901-6.

3. Rothwell PM, Warlow CP. Timing of TIAs preceding stroke: time window for prevention is very short. Neurology 2005; 64(5):817-20, doi:10.1212/01.WNL.0000152985.32732.EE.

4. Gladstone DJ, Kapral MK, Fang J, et al. Management and outcomes of transient ischemic attacks in Ontario. CMAJ 2004;170(7):1099- 104.

5. Rothwell PM, Giles MF, Chandratheva A, et al. Effect of urgent treatment of transient ischaemic attack and minor stroke on early recurrent stroke (EXPRESS study): a prospective population-based sequential comparison. Lancet 2007;370(9596):1432-42, doi:10.1016/S0140-6736(07)61448-2.

6. Coutts SB, Wein TH, Lindsay MP, et al. Canadian Stroke Best Practice Recommendations: secondary prevention of stroke guidelines, update 2014. Int $\mathcal{f}$ Stroke 2015; 282(10):282-91, doi:10.1111/ijs.12439.

7. Canadian Stroke Network. Quality of stroke care in Canada 2011; 2011. Available at: http://www.canadianstro kenetwork.ca/wp-content/uploads/2011/06/QoSC-EN1.pdf (accessed October 2013).

8. Parsons L. Reducing bias in a propensity score matched-pair sample using greedy matching techniques; 2007. Available at: http://www2.sas.com/proceedings/sugi26/p2 14-26.pdf (accessed September 2013).

9. Webster F, Saposnik G, Kapral MK, et al. Organized outpatient care: stroke prevention clinic referrals are associated with reduced mortality after transient ischemic attack and ischemic stroke. Stroke 2011;42(11):3176-82, doi:10.1161/ STROKEAHA.111.621524.

10. Dutta D, Bowen E, Foy C. Four-year follow-up of transient ischemic attacks, strokes, and mimics: a retrospective transient ischemic attack clinic cohort study. Stroke 2015; 46(5):1227-32, doi:10.1161/STROKEAHA.114.008632.

11. Perry JJ, Kerr J, Symington C, et al. How do we manage emergency department patients diagnosed with transient ischemic attack? CFEM 2012;14(1):20-4.

12. Chang E, Holroyd BR, Kochanski P, et al. Adherence to practice guidelines for transient ischemic attacks in an emergency department. Can 7 Neurol Sci 2002; 29(4):358-63.
13. Wu CM, Manns BJ, Hill MD, et al. Rapid evaluation after high-risk TIA is associated with lower stroke risk. Can $\mathcal{F}$ Neurol Sci 2009;36(4):450-5.

14. Kolominsky-Rabas PL, Weber M, Gefeller O, et al. Epidemiology of ischemic stroke subtypes according to TOAST criteria: incidence, recurrence, and long-term survival in ischemic stroke subtypes: a population-based study. Stroke 2001;32(12):2735-40.

15. Palm F, Urbanek C, Wolf J, et al. Etiology, risk factors and sex differences in ischemic stroke in the Ludwigshafen Stroke Study, a population-based stroke registry. Cerebrovasc Dis 2012;33(1):69-75.

16. Blacquiere D, Sharma M, Jetty P. Delays in carotid endarterectomy: the process is the problem. Can 7 Neurol Sci 2013;40(4):585-9.

17. Rothwell PM, Eliasziw M, Gutnikov SA, et al. Endarterectomy for symptomatic carotid stenosis in relation to clinical subgroups and timing of surgery. Lancet 2004;363(9413):915-24, doi:10.1016/S0140-6736(04) 15785-1.

18. Gladstone DJ, Spring M, Dorian P, et al. Atrial fibrillation in patients with cryptogenic stroke. N Engl 7 Med 2014; 370(26):2467-77.

19. Nah H-W, Kwon SU, Kang D-W, et al. Diagnostic and prognostic value of multimodal MRI in transient ischemic attack. Int 7 Stroke 2014;9(7):895-901.

20. Wardlaw J, Brazzelli M, Miranda H, et al. An assessment of the cost-effectiveness of magnetic resonance, including diffusion-weighted imaging, in patients with transient ischaemic attack and minor stroke: a systematic review, meta-analysis and economic evaluation. Health Technol Assess 2014;18(27):1-368; v-vi.

21. Coull AJ, Lovett JK, Rothwell PM. Population based study of early risk of stroke after transient ischaemic attack or minor stroke: implications for public education and organisation of services. BMF 2004;328(7435):326.

22. Giles MF, Rothwell PM. Risk of stroke early after transient ischaemic attack: a systematic review and meta-analysis. Lancet Neurol 2007;6(12):1063-72, doi:10.1016/S14744422(07)70274-0.

23. Moeller JJ, Kurniawan J, Gubitz GJ, et al. Diagnostic accuracy of neurological problems in the emergency department. Can 7 Neurol Sci 2008;35(3):335-41.

24. Prabhakaran S, Silver AJ, Warrior L, et al. Misdiagnosis of transient ischemic attacks in the emergency room. Cerebrovasc Dis 2008;26(6):630-5.

25. Ghia D, Thomas PR, Cordato DJ, et al. Validation of emergency and final diagnosis coding in transient ischemic attack: South Western Sydney transient ischemic attack study. Neuroepidemiology 2010;35(1):53-8. 